\title{
DESIGN OF IMPROVED RESISTOR LESS 45NM SWITCHED INVERTER SCHEME (SIS) ANALOG TO Digital CONVERTER
}

\author{
Arun Kumar Sunaniya ${ }^{1}$ and Kavita Khare $^{2}$ \\ Department of Electronics and Communication Engineering MANIT, Bhopal, India \\ ${ }^{1}$ arun. sunaniya@gmail.com \\ ${ }^{2}$ kavita_khare1@yahoo.co.in
}

\begin{abstract}
This work presents three different approaches which eliminates the resistor ladder completely and hence reduce the power demand drastically of a Analog to Digital Converter. The first approach is Switched Inverter Scheme (SIS) ADC; The test result obtained for it on 45nm technology indicates an offset error of $0.014 \mathrm{LSB}$. The full scale error is of -0.112LSB. The gain error is of $0.07 \mathrm{LSB}$, actual full scale range of $0.49 \mathrm{~V}$, worst case DNL \& INL each of $-0.3 \mathrm{~V}$. The power dissipation for the SIS ADC is $207.987 \mu$ watts; Power delay product (PDP) is $415.9 \mathrm{fWs}$, and the area is $1.89 \mu \mathrm{m} 2$. The second and third approaches are clocked SIS ADC and Sleep transistor SIS ADC. Both of them show significant improvement in power dissipation as $57.5 \%$ \& $71 \%$ respectively. Whereas PDP is $229.7 \mathrm{fWs}$ and area is $0.05 \mu \mathrm{m} 2$ for Clocked SIS $A D C$ and $107.3 \mathrm{fWs} \& 1.94 \mu \mathrm{m} 2$ for Sleep transistor SIS ADC.
\end{abstract}

\section{KEYWORDS}

CMOS 45nm, flash analog to digital converter, low power, resistorless, switched inverter scheme (SIS), sleep transistor.

\section{INTRODUCTION}

Recent advances in the field of electronics require data converters for interfacing the signals of real world for various applications as shown in Fig.1 and then processing the obtained information in digital domain [10], [20]. In present scenario an Analog to Digital Converter (ADC) became an unavoidable component and it is also required to be fabricated on the same chip instead of a separate circuit for data conversion.

Since a few years lot of research is done on data converters to achieve maximum speed and minimum power consumption [8]. As technology scaling continues the demand of minimum power dissipation further increases in various modern battery powered applications [25]. 


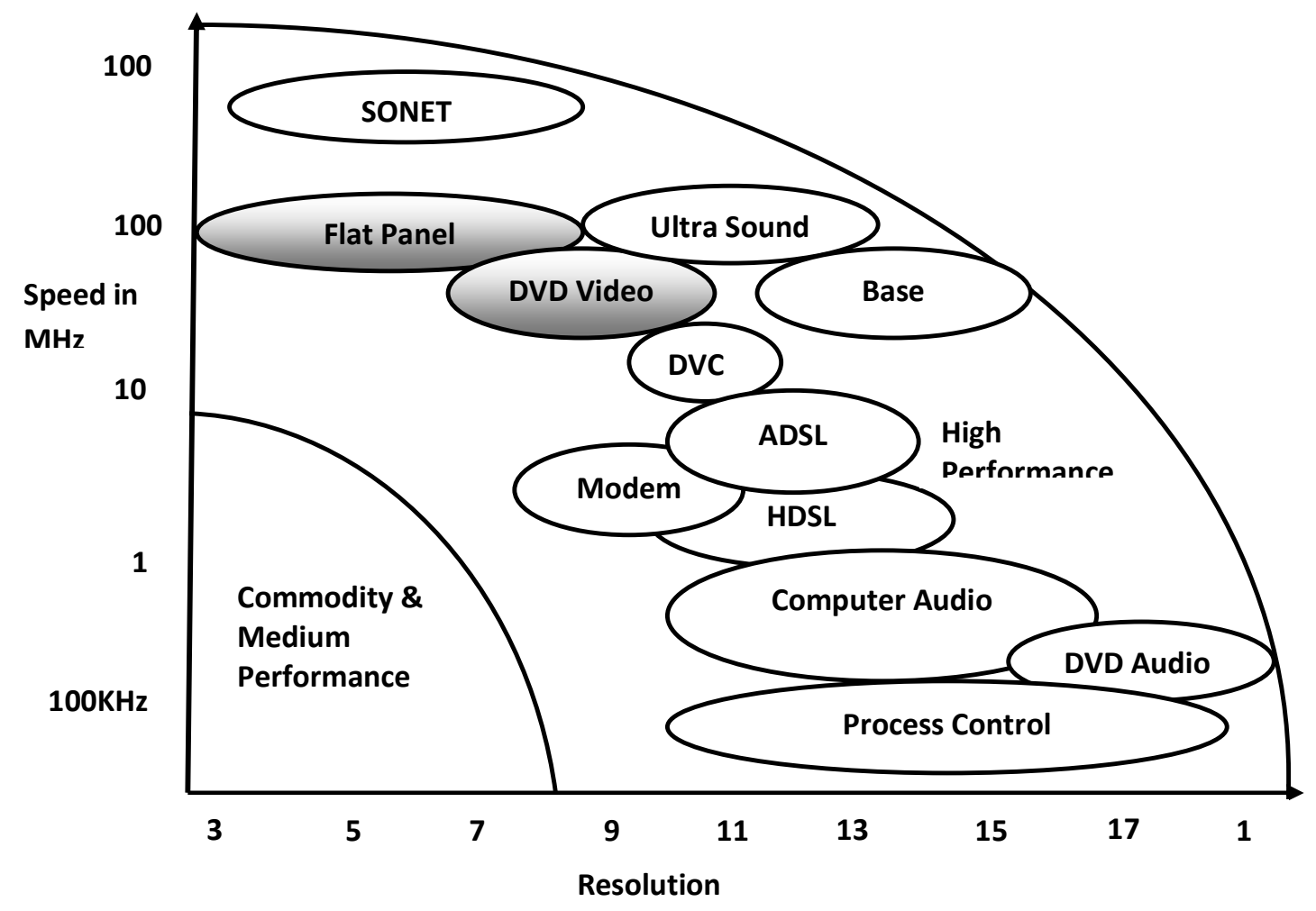

Figure 1. Various Applications of ADC

The research is continuing in various types of data converters like flash, semiflash, sigma delta, pipeline, folding, and successive approximation register (SAR) ADCs to optimize their performance [1], [12]. The insatiable demand of high performance data converters attracts researchers due to their wide usage in digital signal processing. In various applications data converters repress the performance severely if not designed optimally. Investigation of new design techniques for an $\mathrm{ADC}$ is in progress in order to reduce power dissipation, increase operating speed and decrease chip area [3].

High speed data converters are the key building blocks in many applications including high data rate serial links [2], [11]. Ultra wide band systems [4], magnetic and optical data storage devices [5], high speed instrumentation, wideband radar and optical communications [8], 3G telecommunication systems, wireless wide area networks, broadband wireless communication network, radio astronomy, optical communication systems [6]. ADCs with high speed and resolution in the range of 6-8 bits are used in digital telecommunications, cryptography, high performance image sensing and processing, digital signal oscilloscopes, gigabit Ethernet. ADCs with low power dissipation are especially required for system on chip (SOC) applications, cellular phones, Digital TVs, \& spectrum monitoring systems [7].

Out of various $\mathrm{ADC}$ architectures available, the design of flash ADC becomes quite more important as it is used in other ADC architectures like two step ADC and multi bit sigma delta ADC. The block diagram of a flash ADC is as shown in Fig.2 [26], [34]. 


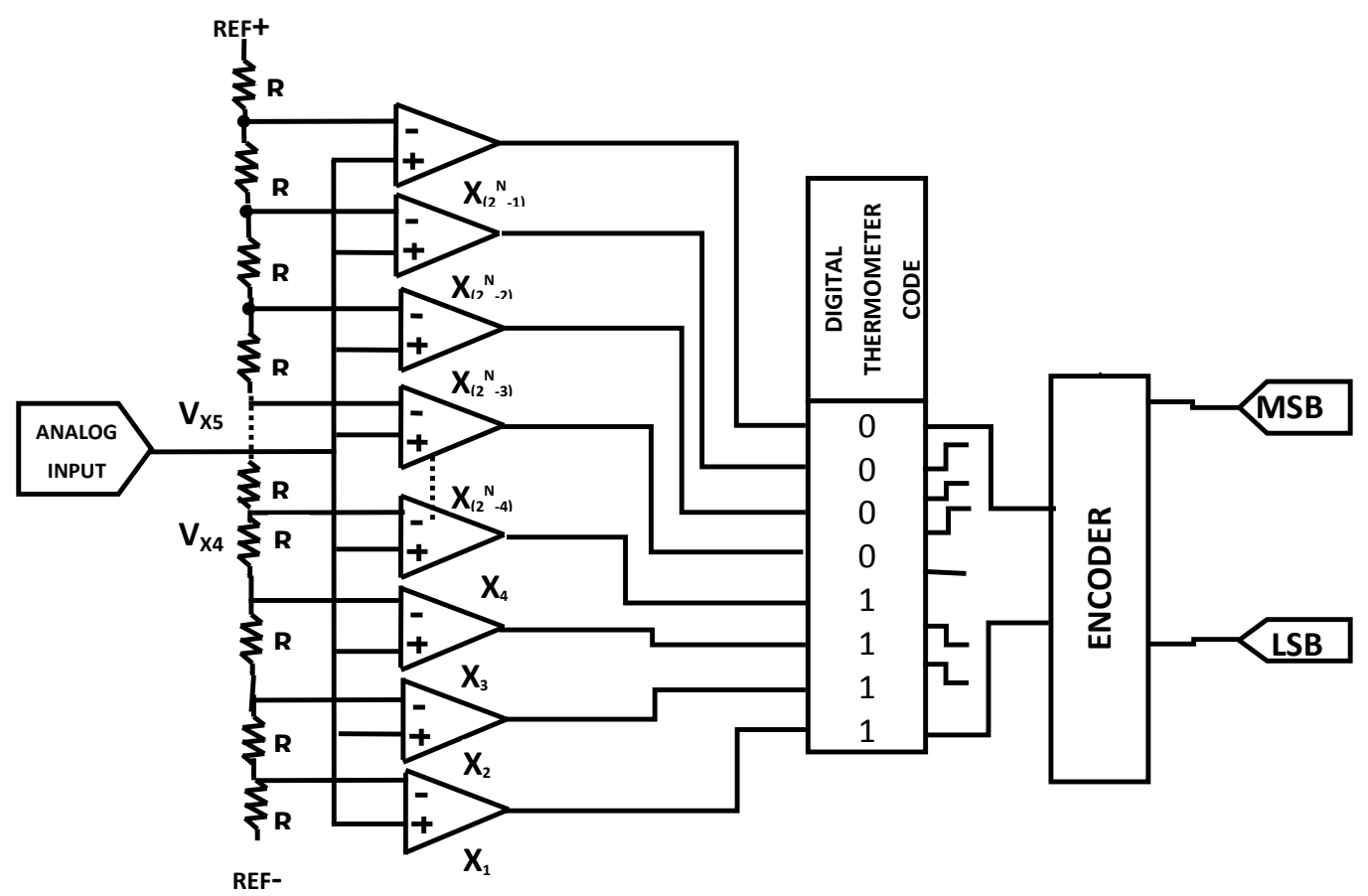

Figure 2. Block Diagram of a Flash ADC

Due to increase in number of resistors and comparators required in designing of fully parallel flash ADCs having resolution 8 bits and above, the layout area \& power dissipation also increases. Therefore in recent years the research is carried with resistor less flash ADC architecture.

New methods developed to reduce the power consumption of flash ADCs are [5].

- Use of interpolation and voltage to current converters that operate as preamplifier stage of latches.

- Extension in the input range.

- Use of bisection method to let only half of the ADCs working in every clock cycle.

The aim of this work is to design the ADCs required for battery operated devices. The ADCs are designed and simulated with three different power saving schemes. The main improvements for various schemes are envisaged in power dissipation, differential and integral non linearity. The specifications of the design prototype are selected as $3 \mathrm{bit}$, for $500 \mathrm{MHz}$ signal, and $0.5 \mathrm{~V}$ full scale range, with a supply voltage of $1 \mathrm{~V}$. The simulation is carried out in 45nm PTM [35] on LT Spice IV platform.

In section II three different types of flash comparator architectures are discussed along with the flow chart and mathematical relations for sizing the transistors of comparator. The design issues of gain booster and encoder blocks are given in section III. Section IV describes the design of ADC using all three comparators. The complete simulation results are shown in section V. Section VI concludes the work. 
International Journal of VLSI design \& Communication Systems (VLSICS) Vol.4, No.3, June 2013

\section{DIFFERENT TYPES OF FLASH COMPARATORS}

\subsection{Switched Inverter Scheme (SIS) Comparator}

When focusing on overall power for a flash ADC, the power dissipation of the comparator is important contributor. In case of $n$ bit flash converters the number of comparator equals $2^{n}-1$ [39]. The switched inverter scheme (SIS) also called Threshold inverter quantization (TIQ) comparator has very simple architecture as shown in Fig.3 [37], [38]. It is quite different than the conventional operational amplifier based differential input voltage (DIV) comparator [9], [28], [34]. The key difference between the DIV comparator and the (SIS) comparator scheme lies in the way the reference voltage is generated for each level. In DIV comparator conventional method of using resistor ladder is utilized for externally generating the reference voltage. Where as in the SIS comparator scheme all $2^{\mathrm{n}}-1$ reference voltage for a $\mathrm{n}$ bit ADC are set internally by adjusting the threshold voltage of each voltage comparator separately by sizing the transistor properly. All DIV comparators are identical and duplicated for $2^{\mathrm{n}}-1$ times but each SIS comparator is altogether different from others and obtained by varying the $\beta$ ratio of the inverters.

The SIS comparator design consists of two pairs of inverters connected back to back. Each of the inverter is sized separately to get a unique switching voltage [13], [17].

The cascaded inverters then work as voltage comparator. The full scale voltage range (VFSR) is equally divided by $2^{\mathrm{n}}-1$ SIS comparators.

To achieve optimal sizing for each transistor in SIS comparator scheme is very much time consuming. The analytical expression for $\left(\mathrm{W}_{\mathrm{p}} / \mathrm{W}_{\mathrm{n}}\right)$ of each comparator is determined by equating the pull up and pull down saturation currents as equation (1) [14], [15], [16], [18].

$$
\mathrm{I}_{\mathrm{DSAT}}=\mathrm{K} \cdot \mathrm{V}_{\mathrm{DSAT}}\left(\mathrm{V}_{\mathrm{m}}-\mathrm{V}_{\mathrm{DD}}-\mathrm{V}_{\mathrm{t}}-\frac{\mathrm{V}_{\mathrm{DSAT}}}{2}\right)
$$

The expression for switching voltage $\mathrm{V}_{\mathrm{m}}$ is thus obtained for a short channel CMOS inverter in 45nm as equation (2) [19], [21], [22], [29].

$$
\mathrm{V}_{\mathrm{m}}=\frac{\mathrm{V}_{\mathrm{DD}}-\left|\mathrm{V}_{\mathrm{tp}}\right|+\mathrm{V}_{\mathrm{tn}} \sqrt{1 / \mathrm{r}}}{1+\sqrt{1 / \mathrm{r}}}
$$

Where,

$$
\begin{aligned}
& r=\frac{v_{\text {satp }} W_{p}}{v_{\text {satn }} W_{n}}=\frac{K_{p} V_{\text {DSATp }}}{K_{n} V_{\text {DSATn }}} \\
& r=\frac{\mu_{p}\left(W_{p} / L_{p}\right)}{\mu_{n}\left(W_{n} / L_{n}\right)} \\
& K_{p}=K_{p}^{\prime}\left(\frac{W_{p}}{L_{p}}\right)=\beta_{p}
\end{aligned}
$$




$$
\begin{aligned}
& \mathrm{K}_{\mathrm{p}}^{\prime}=\mu_{\mathrm{p}} \mathrm{C}_{\mathrm{oxp}} \\
& \mathrm{K}_{\mathrm{n}}=\mathrm{K}_{\mathrm{n}}^{\prime}\left(\frac{\mathrm{W}_{\mathrm{n}}}{\mathrm{L}_{\mathrm{n}}}\right)=\beta_{\mathrm{n}} \\
& \mathrm{K}_{\mathrm{n}}^{\prime}=\mu_{\mathrm{n}} \mathrm{C}_{\mathrm{oxn}}
\end{aligned}
$$

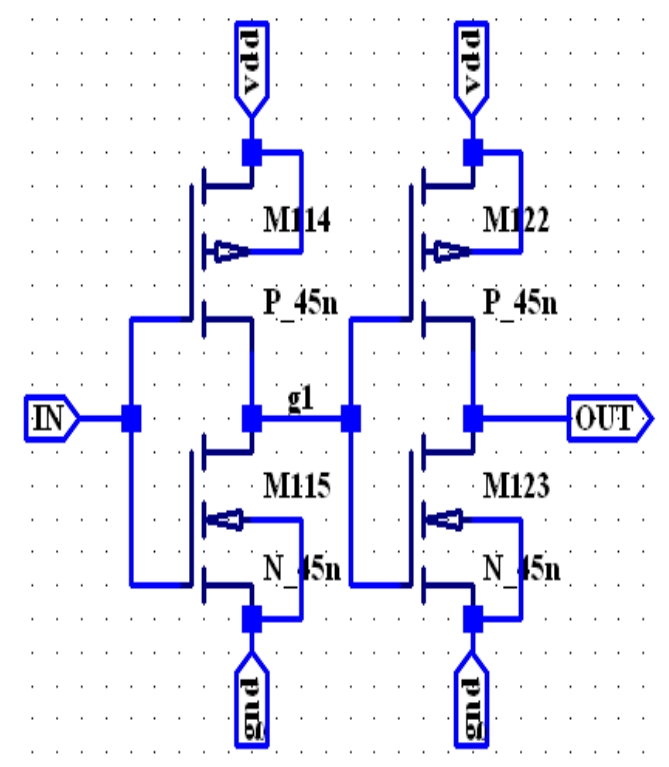

Figure 3. SIS Comparator

The above equations are realized on MATLAB 7.3.0 R2006b or switching voltage calculations. The program is given in Fig.4.

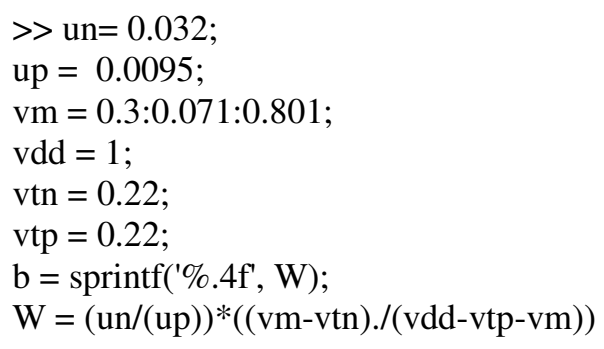

Figure 4. MATLAB code for switching voltage calculation

The main advantage of the SIS comparators is that it does not use any resistor ladder and so it is suitable for low voltage, low power and high speed applications such as cellular mobile phones and touch screen display devices. On the other hand the process parameter variations may cause offset and gain errors to vary considerably when the design is used for higher resolution ADCs. Also noise can be a big challenge for the single ended SIS comparator scheme. 


\subsection{Clocked SIS comparator}

Another type of the comparator as shown in Fig.5.called as clocked SIS comparator (CSIS) uses two cascaded inverters as SIS and two set of PMOS and NMOS connected in parallel. Each pair connected to pull up and pull down networks of the SIS inverter. The pair of PMOS and NMOS is driven by a clock pulse the NMOS is connected to clock whereas PMOS to clockbar. The two pairs are sized to minimum length and width in $45 \mathrm{~nm}$ technology to get reduction in static power dissipation of the overall voltage comparator. The saving in power dissipation with the modification is approximately $60.37 \%$ to that of the SIS comparator.

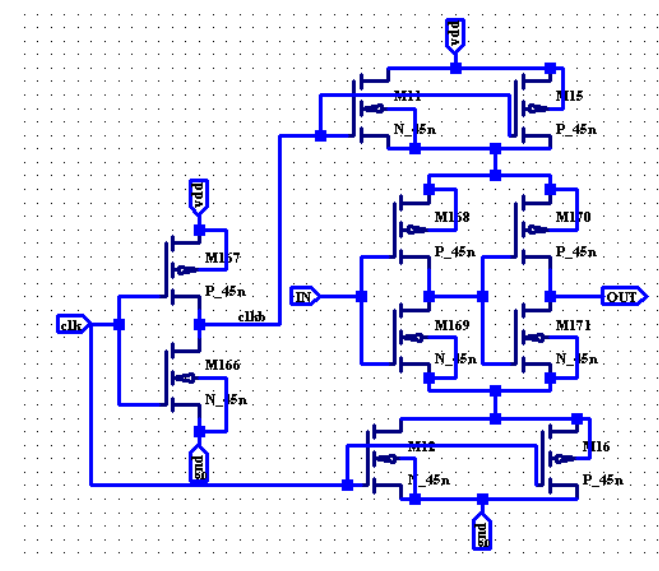

Figure 5. Clocked SIS Comparator

\subsection{SIS comparator with sleep transistor}

The comparison circuit of SIS comparator is modified by addition of high threshold PMOS and NMOS near the supply rails as shown in Fig.6. The addition of header \& footer reduces the static power dissipation to a great extent due to increased resistance of the high threshold PMOS and NMOS transistors [27], [30], [33]. During the period of no activity the section not in use remains completely off. Whereas, the components are invoked again when any activity is detected.

A Local sleep transistor network is used here as opposed to global level network, because every comparator is differently sized and hence the current through each comparator section is also different. The sizing of a sleep transistor is calculated using equation (9).

$$
\left(\frac{\mathrm{W}}{\mathrm{L}}\right)_{\text {Sleep }}=\frac{\mathrm{I}_{\text {Sleep }}}{0.0281 \mu_{\mathrm{n}} \mathrm{C}_{\mathrm{OX}}\left(\mathrm{V}_{\mathrm{DD}}-\mathrm{V}_{\mathrm{T}}\right)\left(\mathrm{V}_{\mathrm{DD}}-\mathrm{V}_{\mathrm{TH}}\right)}(9)
$$

Where, $\mathrm{I}_{\text {sleep }}$ is calculated by simulating the circuit without sleep transistor network and finding maximum current that flows through ground or $\mathrm{V}_{\mathrm{DD}}$. $\mathrm{V}_{\mathrm{T}}$ denotes threshold voltages for short channel device \& $\mathrm{V}_{\mathrm{TH}}$ is equal to threshold voltage of high $\mathrm{K}$ device.

$$
\begin{aligned}
& \mathrm{C}_{\mathrm{ox}}^{\prime}=\frac{\epsilon_{\mathrm{ox}}}{\mathrm{t}_{0 \mathrm{x}}} \\
& \mathrm{C}_{\mathrm{ox}}=\frac{\epsilon_{0} \cdot \epsilon_{\mathrm{ox}}}{\mathrm{t}_{0 \mathrm{x}}}
\end{aligned}
$$




$$
\mathrm{C}_{\mathrm{ox}}=\mathrm{C}_{\mathrm{ox}}^{\prime} \cdot \mathrm{A}
$$

$$
\mathrm{A}=\mathrm{W} \cdot \mathrm{L}
$$

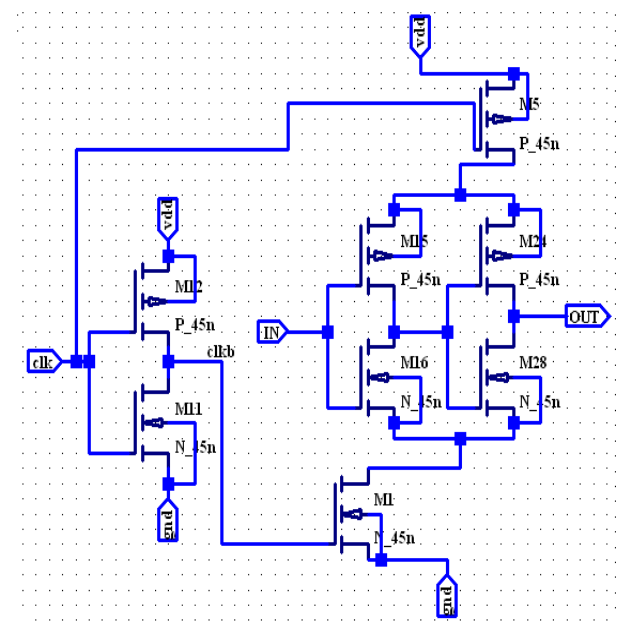

Figure 6. SIS Comparator with Sleep Transistor

The saving in power dissipation with the addition of header \& footer is approximately $77.3 \%$ to that of the SIS comparator.

\section{DESIGN OF ADCS}

For the complete realization of any flash ADC the blocks required other than comparator are gain booster and thermometer to binary encoder. The design of these blocks is given here.

\subsection{Gain Booster}

The gain booster is used to increase voltage gain of the output of a comparator so that it provides a full digital output voltage swing, without which the output of the comparator circuit is unable to drive the next stage, it also makes thresholds sharper for comparator outputs and provide full digital output voltage swing. The gain booster block is designed for lowest switching voltage.

\begin{tabular}{|c|c|c|c|c|c|c|c|c|c|c|c|c|c|}
\hline \multicolumn{7}{|c|}{ Input Thermometer code } & \multicolumn{7}{|c|}{ Output "01" code } \\
\hline $\mathrm{T}_{7}$ & $\mathrm{~T}_{6}$ & $\mathrm{~T}_{5}$ & $\mathrm{~T}_{4}$ & $\mathrm{~T}_{3}$ & $\mathrm{~T}_{2}$ & $\mathrm{~T}_{1}$ & $\mathrm{G}_{7}$ & $\mathrm{G}_{6}$ & $\mathrm{G}_{5}$ & $\mathrm{G}_{4}$ & $\mathrm{G}_{3}$ & $\overline{\mathrm{G}_{2}}$ & $\overline{\mathrm{G}_{1}}$ \\
\hline 0 & 0 & 0 & 0 & 0 & 0 & 0 & 0 & 0 & 0 & 0 & 0 & 0 & 1 \\
\hline 0 & 0 & 0 & 0 & 0 & 0 & 1 & 0 & 0 & 0 & 0 & 0 & 1 & 0 \\
\hline 0 & 0 & 0 & 0 & 0 & 1 & 1 & 0 & 0 & 0 & 0 & 1 & 0 & 0 \\
\hline 0 & 0 & 0 & 0 & 1 & 1 & 1 & 0 & 0 & 0 & 1 & 0 & 0 & 0 \\
\hline 0 & 0 & 0 & 1 & 1 & 1 & 1 & 0 & 0 & 1 & 0 & 0 & 0 & 0 \\
\hline
\end{tabular}

Table 1. Truth table for one out of $n$ code 
International Journal of VLSI design \& Communication Systems (VLSICS) Vol.4, No.3, June 2013

\begin{tabular}{|c|c|c|c|c|c|c|c|c|c|c|c|c|c|}
\hline 0 & 0 & 1 & 1 & 1 & 1 & 1 & 0 & 1 & 0 & 0 & 0 & 0 & 0 \\
\hline 0 & 1 & 1 & 1 & 1 & 1 & 1 & 1 & 0 & 0 & 0 & 0 & 0 & 0 \\
\hline 1 & 1 & 1 & 1 & 1 & 1 & 1 & 0 & 0 & 0 & 0 & 0 & 0 & 0 \\
\hline
\end{tabular}

Each gain booster consists of two identical cascaded inverters with the same circuit as that of comparator, but the transistors are sized for minimum aspect ratio for each gain booster [23].

\subsubsection{Thermometer code converter}

The encoder converts the thermometer code to binary code in two steps [40]. In the first step the thermometer code is converted into one out of $\mathrm{n}$ code by using the truth table as shown in Table 1. The one out of $n$ codes is then converted to binary code $d 2, d 1$, d0 by Read only memory (ROM) encoder, as shown in Fig.7. [32]. The ROM encoder is a common and straight forward approach to encode the one out of $n$ code to binary bit. The appropriate row $m$ in the ROM is selected by using a row decoder that has the output of comparator $\mathrm{m}$ and the inverse of comparator $m+1$ as inputs. The output $m$ of the row decoder, connected to memory row $m$, is high if the output of comparator $m$ is high and the output of comparator $m+1$ is low. The row decoder can be realized by, a number of 2-input NAND gates, where one input to each NAND gate is inverted. The main advantage of the ROM decoder approach is its regular structure that is easy to design [31].

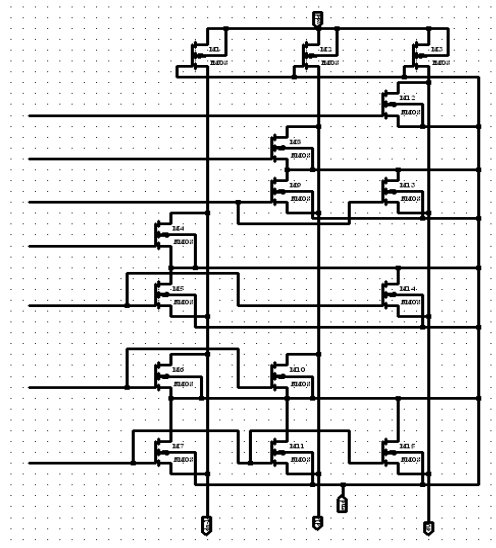

Figure 7. ROM Encoder

\section{RESULTS AND DISCUSSION}

The proposed technique is applied in the design of 3 bit ADC with chosen specifications as given in table II and the implementation is as shown in Fig.8. for SIS ADC, Fig.10. for Clocked SIS ADC \& in Fig.12. for sleep transistor ADC [23], [24].

The design consists of comparator chain, gain booster circuit and a thermometer to binary encoder block and finally a buffer circuitry to reduce the loading effects.

Conventional voltage comparators use a resistor ladder circuit to generate $2^{\mathrm{n}}$-1reference voltages $\left(\mathrm{V}_{\text {ref }}\right)$ for comparators, ranging from $\mathrm{V}_{\text {ref(max) }}$ to $\mathrm{V}_{\text {ref(min) }}$. Generating equally spaced reference voltages determines the quantization performance of ADCs [21]. The SIS comparator eliminates the use of the resistor ladder circuit by generating the reference voltages internally. The SIS comparator uses two cascaded inverters to generate the range of internal reference voltages. At 
International Journal of VLSI design \& Communication Systems (VLSICS) Vol.4, No.3, June 2013

the first inverter, the analog input quantization is determined by adjusting the ratio of the PMOS and PMOS transistors. The second inverter is used to increase the voltage gain and prevent unbalanced propagation delays of all comparators.

The results for SIS Comparator are shown in Fig. 8. and for the modified versions 2 and 3 they are given in Fig.11. and Fig.13.

The total power consumption here is determined by summing up the power dissipation of each circuit separately. The various blocks used to realize the design are comparator section, gain booster, XOR gate, ROM Encoder and buffers the comparison results are shown in table III for all three different types of ADCs [9], [17], [30], [37], [38].

Table 2. ADC Specification

\begin{tabular}{|c|c|c|}
\hline S No. & Specification & Value \\
\hline 1 & Resolution & $3 \mathrm{Bit}$ \\
\hline 2 & CMOS tech. & $45 \mathrm{~nm}$ \\
\hline 3 & Model File & PTM \\
\hline 4 & $\mathrm{~L}_{\mathrm{P}}=\mathrm{L}_{\mathrm{N}}=\mathrm{L}_{\min }$ & $45 \mathrm{~nm}$ \\
\hline 5 & $t_{\mathrm{ox}}$ & 14 Angstroms \\
\hline 6 & $\mathrm{C}_{\mathrm{ox}}$ & $25 \mathrm{fF} / \mu \mathrm{m}^{2}$ \\
\hline 7 & Max. Speed & 500 MSPS \\
\hline 8 & $\mathrm{~V}_{\mathrm{FSR}}$ & $0.3 \mathrm{~V}-0.8 \mathrm{~V}$ \\
\hline 9 & $\mathrm{~V}_{\mathrm{LSB}}$ & $0.0714 \mathrm{~V}$ \\
\hline 10 & $\left(\mathrm{~W}_{\mathrm{p}} / \mathrm{W}_{\mathrm{n}}\right)_{\mathrm{Min}}$ & 0.55 \\
\hline 11 & $\left(\mathrm{~W}_{\mathrm{p}} / \mathrm{W}_{\mathrm{n}}\right)_{\mathrm{Max}}$ & 266 \\
\hline 12 & $\mathrm{~V}_{\mathrm{DD}}$ & $1 \mathrm{~V}$ \\
\hline 13 & $\mathrm{~V}_{\mathrm{TP}}$ & $-0.22 \mathrm{~V}$ \\
\hline 14 & $\mathrm{~V}_{\mathrm{TN}}$ & $0.22 \mathrm{~V}$ \\
\hline 15 & $\mathrm{~V}_{\mathrm{m} \min }$ & $0.371 \mathrm{~V}$ \\
\hline 16 & $\mathrm{~V}_{\mathrm{m} \max }$ & $0.8 \mathrm{~V}$ \\
\hline 17 & $\mu_{n}$ & $320 \mathrm{~cm}^{2} / \mathrm{v}$-sec \\
\hline 18 & $\mu_{p}$ & $95 \mathrm{~cm}^{2} / \mathrm{v}$-sec \\
\hline 19 & $\mathrm{C}_{\mathrm{L}}($ Load Capacitance $)$ & $0.01 \mathrm{fF}$ \\
\hline
\end{tabular}


International Journal of VLSI design \& Communication Systems (VLSICS) Vol.4, No.3, June 2013

\subsection{Design of SIS ADC}

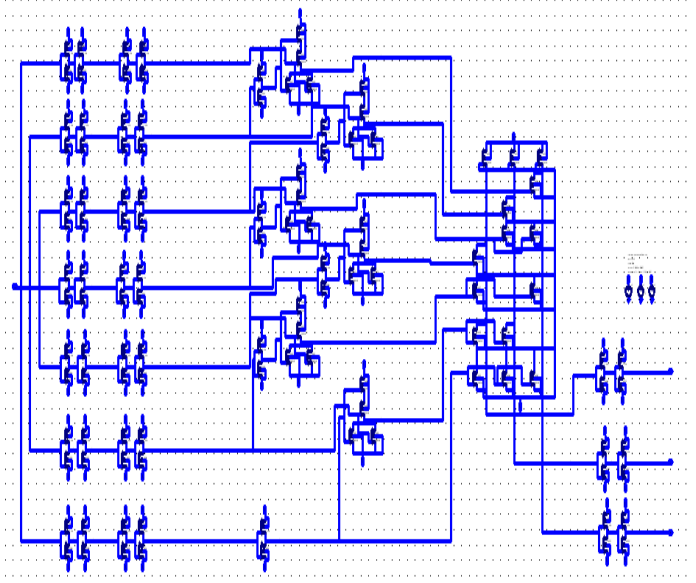

Figure 8. SIS ADC with ROM Encoder

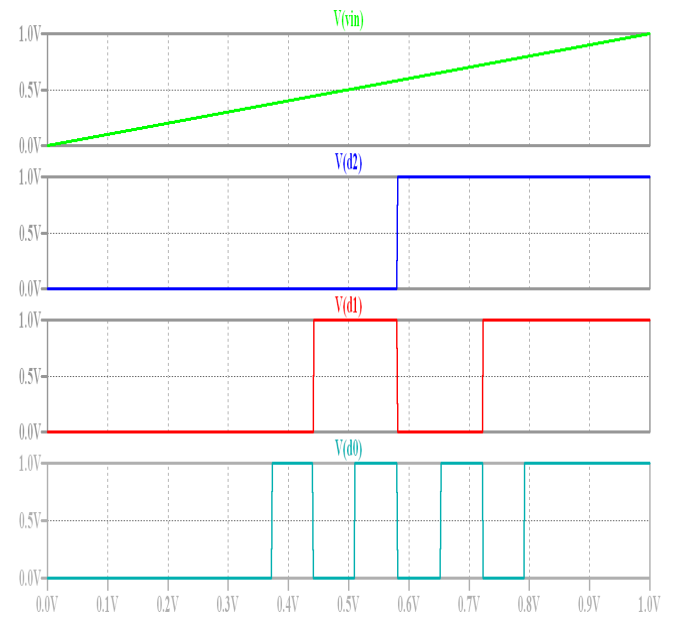

Figure 9. Simulation Results of SIS ADC

\subsection{Design of Clocked SIS ADC}

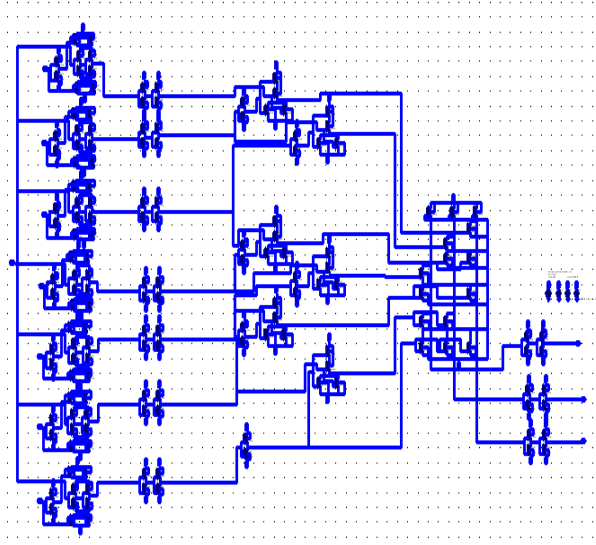

Figure 10. Clocked SIS ADC with ROM Encoder 
International Journal of VLSI design \& Communication Systems (VLSICS) Vol.4, No.3, June 2013

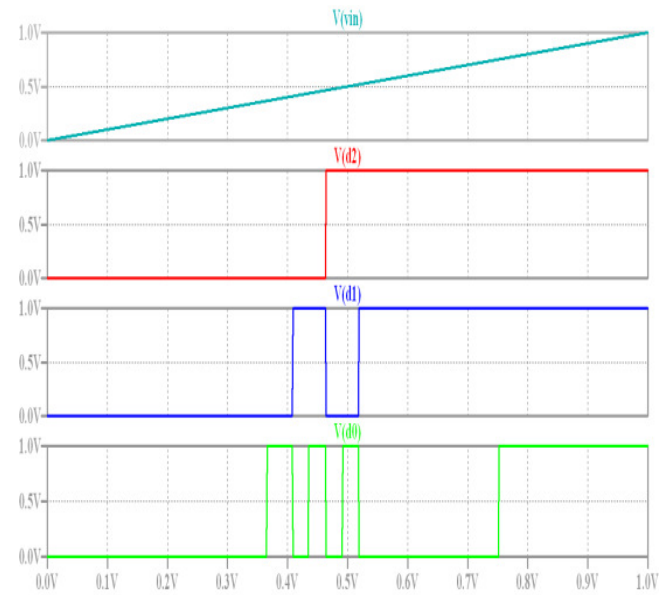

Figure 11. Simulation Results of Clocked SIS ADC

\subsection{Design of Sleep Transistor SIS ADC}

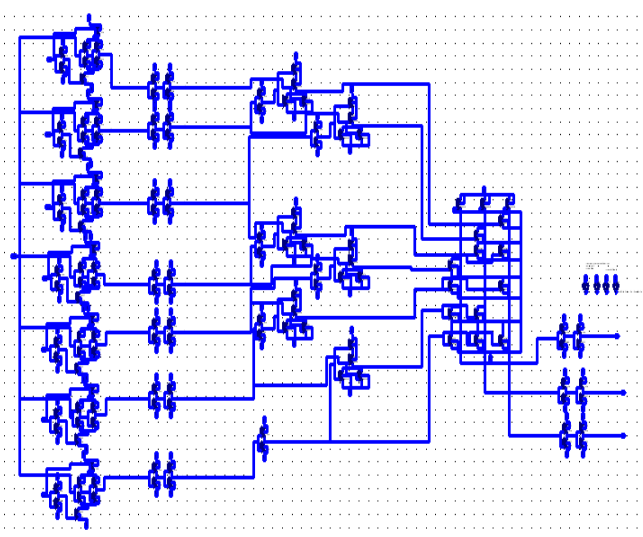

Figure 12. Sleep Transistor SIS ADC with ROM Encoder

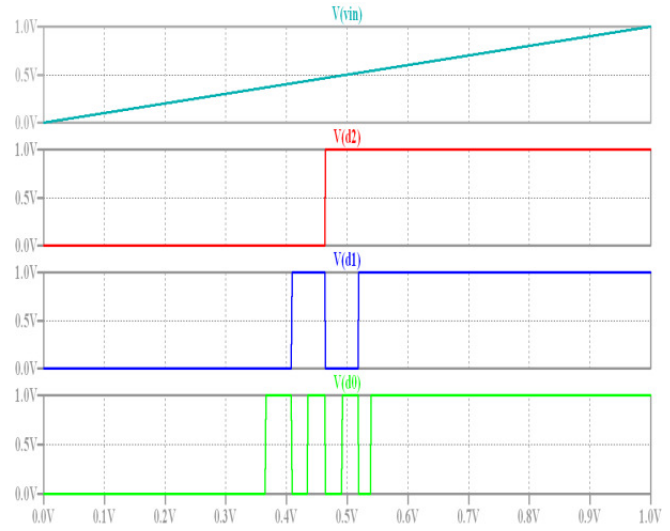

Figure 13. Simulation Results of Sleep Transistor SIS ADC

The power dissipation is calculated and is compared for all the designed ADCs. The finding for saving in power dissipation is shown in Table IV. 
International Journal of VLSI design \& Communication Systems (VLSICS) Vol.4, No.3, June 2013

The various dynamic parameters for all ADCs are also calculated using the equations (15) - (21) [29], [36] and are tabulated in Table V.

Offset error $=$ Real Transition - Ideal Transition

Offset in LSB = Offset Error/ One LSB

Full Scale Error $=$ Real last transition - Ideal last transition

Gain error $=$ Average code width $($ LSB size $)$

$=$ Real last transition - First transition $/ 2\left(2^{\mathrm{n}}-1-1\right)$

$\mathrm{DNL}=\mathrm{DNL}[\mathrm{k}]=$ Width $[\mathrm{k}]-1$

Where,

Width $[\mathrm{k}]=$ Transition $[\mathrm{k}+1]-$ Transition $[\mathrm{k}]$

INL $(\mathrm{m})=$ Transition [m] - Transition[ideal] / Step Width [Ideal] (21)

Table 3. Comparison Of Component Power Dissipation In $\mu \mathrm{W}$

\begin{tabular}{|c|l|c|c|c|}
\hline S.No. & \multicolumn{1}{|c|}{ Blocks } & SIS ADC & Clocked SIS ADC & Sleep SIS ADC \\
\hline 1 & Comparator & 167.087 & 66.214 & 37.88 \\
\hline 2 & Gain Booster & 35.18 & 18.678 & 18.48 \\
\hline 3 & One out of n coder & 3.1357 & 1.9 & 1.8 \\
\hline 4 & ROM encoder & 2.153 & 1.1 & 1.09 \\
\hline 5 & Buffers & 0.437 & 0.32 & 0.31 \\
\hline 6 & Total Power & 207.987 & 88.377 & 59.64 \\
\hline 7 & Delay & $2 \mathrm{~ns}$ & $2.6 \mathrm{~ns}$ & $1.8 \mathrm{~ns}$ \\
\hline 8 & Power Delay Product & $415.9 \mathrm{fWs}^{-18}$ & $229.7 \mathrm{fWs}$ & $107.3 \mathrm{fWS}$ \\
\hline 9 & Area & $1.89 \mu \mathrm{m}^{2}$ & $2.05 \mu \mathrm{m}^{2}$ & $1.94 \mu \mathrm{m}^{2}$ \\
\hline
\end{tabular}

$$
\mathrm{INL}=\sum_{\mathrm{i}=1}^{\mathrm{m}} \mathrm{DNL}[\mathrm{i}]
$$

DNL is Differential non linearity error and INL is Integral non linearity error. The quantization error in each case is less than 0.5 LSB. 
International Journal of VLSI design \& Communication Systems (VLSICS) Vol.4, No.3, June 2013

Table 4. Block wise \% saving in power dissipation as compared to SIS ADC

\begin{tabular}{|c|c|c|c|}
\hline S.No. & Blocks & $\begin{array}{c}\text { Clocked SIS ADC } \\
{[\mathbf{1 7}],[30],[37],[38]}\end{array}$ & Sleep SIS ADC \\
\hline 1 & Comparator & $60.37 \%$ & $77.3 \%$ \\
\hline 2 & Gain Booster & $46.9 \%$ & $47.47 \%$ \\
\hline 3 & One out of n coder & $39.4 \%$ & $42.6 \%$ \\
\hline 4 & ROM encoder & $48.9 \%$ & $49.37 \%$ \\
\hline 5 & Buffers & $26.77 \%$ & $29.06 \%$ \\
\hline 6 & Total Power & $57.5 \%$ & $71 \%$ \\
\hline
\end{tabular}

Table 5. Dynamic Parameters For ADCs In LSB

\begin{tabular}{|c|c|c|c|}
\hline Parameter & SIS ADC & Clocked SIS ADC & Sleep Transistor SIS ADC \\
\hline Offset Error & 0.014 & 0.158 & -0.084 \\
\hline Full Scale Error & -0.112 & -0.26 & -0.36 \\
\hline Gain Error & 0.07 & 0.028 & 0.021 \\
\hline DNL & -0.3 & -0.7 & -0.6 \\
\hline INL & -0.3 & -0.7 & -0.4 \\
\hline
\end{tabular}

\section{CONCLUSION}

Low power architecture for a 3-bit CMOS SIS based flash ADC is presented using PTM $45 \mathrm{~nm}$. The modified versions of SIS ADC can further achieve very low power dissipation; this proposed method can reduce power dissipation upto $71 \%$. It uses smaller silicon area of $1.94 \mu \mathrm{m} 2$. The DNL of the proposed ADC is within -0.3LSB and INL is also within -0.3LSB. The proposed ADC chip so designed can be used for capacitive pressure sensor, video systems as well as it can be used in a low power two-step ADC, pipelined ADC and multi-bit sigma delta ADC. A disadvantage is that for higher no of bits the increased complexity of the circuit consumes more chip area and will likely consume more power.

\section{REFERENCES}

[1] Amol Inamdar, Anubhav Sahu, Jie Ren, Aniruddha Dayalu, and Deepnarayan Gupta, (2013) "Flash ADC Comparators and Techniques for their Evaluation", IEEE Transactions on Applied Superconductivity, Vol.23, no.3, ISSN No.1051-8223, pp. 1-8.

[2] Xiangliang Jin, Zhibi Liu, and Jun Yang, (2013) "New Flash ADC Scheme With Maximal 13 Bit Variable Resolution and Reduced Clipped Noise for High-Performance Imaging Sensor", IEEE Sensors Journal, Vol. 13, no.1, pp. 167-171.

[3] Young-Kyun Cho, Jae-Ho Jung, and Kwang Chun Lee, (2012) "A 9-bit 100-MS/s Flash-SAR ADC without Track-and-Hold Circuits”, International Symposium on Wireless Communication Systems (ISWCS), ISSN: 2154-0217, ISBN No. 978-1-4673-0761-1, pp.880-884.

[4] Joyjit Mukhopadhyay and Soumya Pandit, (2012) “Modeling and Design of a Nano Scale CMOS Inverter for Symmetric Switching Characteristics”, Hindawi Publishing Corporation VLSI Design, Vol.2012, pp.1-13. 
International Journal of VLSI design \& Communication Systems (VLSICS) Vol.4, No.3, June 2013

[5] Chakir Mostafa, Hassan Qjidaa, (2012) "1 GS/s, Low Power Flash Analog to Digital Converter in 90nm CMOS Technology", IEEE International conference on Multimedia Computing and Systems (ICMCS), 2012, ISBN: 978-1-4673-1518-0, pp.1097 - 1100.

[6] Yun-Shiang Shu, (2012) "A 6b 3GS/s 11mW Fully Dynamic Flash ADC in 40nm CMOS with Reduced Number of Comparators", IEEE Symposium on VLSI Circuits Design, ISBN: 978-1-46730848-9, pp. 26-27.

[7] Ch. Vassou, L. Mountrichas, S. Siskos, (2012) "A NMOS Bulk Voltage Trimming Offset Calibration Technique for a 6-bit 5GS/s Flash ADC”, IEEE International Conference on Instrumentation and Measurement Technology (I2MTC), ISSN: 1091-5281 ISBN: 978-1-4577-1773-4, pp.5-8.

[8] Soon-Kyun Shin, Jacques C. Rudell, Denis C. Daly, Carlos E. Muñoz, Dong-Young Chang, Kush Gulati, Hae-Seung Lee, and Matthew Z. Straayer, (2012)“A 12b 200MS/s Frequency Scalable ZeroCrossing Based Pipelined ADC in 55nm CMOS" ,IEEE Custom Integrated Circuits Conference (CICC), ISSN: 0886-5930 978-1-4673-1555-5, pp.1-4.

[9] Yuji Gendai, and Akira Matsuzawa, (2012) "A Specific Distortion Pattern of Flash ADCs Identified by Discriminating Time-Domain Analysis", IEEE Transactions on instrumentation and measurement, vol. 61, no. 2, pp.316-325.

[10] Amir Zjajo Jose, Pineda de Gyvez (2011) "Low-Power High Resolution Analog to Digital Converters, Design Test and Calibration" ISBN 978-90-481-9724-8, First edition, Springer New York.

[11] Dharmendra Mani Varma (2011)“Reduced Comparator Low power Flash ADC using 35nm CMOS”, IEEE Conference on Electronics Computer Technology (ICECT), Vol.1, pp. 385- 388.

[12] Pierluigi Nuzzo, Claudio Nani, Costantino Armiento, Alberto Sangiovanni Vincentelli, Jan Craninckx, Geert Van der Plas,(2011) “A 6-Bit 50-MS/s Threshold Configuring SAR ADC in 90-nm Digital CMOS”,IEEE Transactions On Circuits And Systems-I, Vol.59, no. 1. pp. 80-92.

[13] Sudakar S. Chauhan, S. Manabala, S.C. Bose and R. Chandel,(2011) "A New Approach To Design Low Power CMOS Flash A/D Converter", International Journal of VLSI design \& Communication Systems(VLSICS),Vol.2, no.2,pp.10-108.

[14] A. Ávila, D. Espejo,(2011) "A SPICE-compatible Model for Intel's 45nm High K MOSFET", The World Congress on Engineering and Computer Science (WCECS) Proceedings, ISBN no.978-98819251-7-6, ISSN: 2078-0958, Vol.2, pp.762-765.

[15] G. Torfs, Z. Li, J. Bauwelinck, X. Yin, G. Van der Plas and J.Vandewege, "Low-power 4-bit flash analogue to digital converter for ranging applications", IEEE Electronics Letters, Vol. 47 no.1, pp 2022.

[16] T. Esther Rani, Dr. Rameshwar rao, (2011) "Area And Power Optimized Multipliers With Minimum Leakage", IEEE International Conference on Electronic Computer Technology - ICECT , ISBN no.978-1-4244-8679. Vol. 3, pp.284 -287.

[17] Oktay Aytar and Ali Tangel, (2011) "Employing threshold inverter quantization (TIQ) technique in designing 9-Bit folding and interpolation CMOS analog-to-digital converters (ADC)", Scientific Research and Essays,Vol.6,no.2, ISSN no. 1992-2248, pp. 351-362.

[18] Hiroshi Fuketa, Satoshilida, Tadashi Yasufuku, Makoto Takamiya, Masahiro Nomura, Hirofumi Shinohara, Takayasu Sakurai,(2011) "A Closed-form Expression for Estimating Minimum Operating Voltage (VDDmin) of CMOS Logic Gates", IEEE Conference on Design Automation (DAC), ISSN: 0738-100x, ISBN: 978-1-4503-0636-2, pp. 984-989.

[19] Baozeng Guo, Tao Ma, Yubo Zhang, (2011) "Design of A Novel Domino XNOR Gate for 32-nm node CMOS Technology”, IEEE International conference on Electric Information and Control Engineering (ICEICE), ISBN no. 978-1-4244-8036-4, pp. 289-292.

[20] Tomoyuki Yamase, Hiroaki Uchida, and Hidemi Noguchi,(2011)“A 22-mW 7b 1.3-GS/s Pipeline ADC with 1-bit/stage Folding Converter Architecture”, Symposium on VLSI Circuits Digest of Technical Papers, ISSN no. 2158-5601, ISBN no. 978-4-86348-1657, pp.124-125.

[21] Jaeyoon Kim, Sandip Tiwari, (2011) "Inexact Computing for Ultra Low Power Nanometer Digital Circuit Design”, IEEE/ACM International Symposium on Nanoscale Architectures, ISBN no. 978-14577-0995-1, pp.24-31.

[22] Tsung-Ching Huang, Kenjiro Fukuda, Chun-Ming Lo, Yung-Hui Yeh, Tsuyoshi Sekitani, Takao Someya and Kwang-Ting Cheng,(2011) "Pseudo-CMOS: A Design Style for Low-Cost and Robust Flexible Electronics" IEEE Transactions on Electron Devices, Vol. 58, no.1, pp. 141-150.

[23] Arun Kumar Sunaniya, Kavita Khare,(2011) "A Design Comparison of Low Power $50 \mathrm{~nm}$ Technology Based Inverter with Sleep Transistor and MTCMOS Scheme", International Journal of Engineering Science and Technology (IJEST), Vol. 3 No. 10, ISSN no. 0975-5462,pp. 7744-7753. 
International Journal of VLSI design \& Communication Systems (VLSICS) Vol.4, No.3, June 2013

[24] Arun Kumar Sunaniya, Kavita Khare, (2011)“A Low power 50 nm Technology Based CMOS Inverter with Sleep Transistor Scheme” International Journal of Computer Science Engineering \& Technology (IJCSET), Vol. 1 No. 9, ISSN no. 2231-0711,pp. 560-562.

[25] The (2010) "International Technology Road map of semiconductor" ITRS, pp 61-66.

[26] Meghana Kulkarni, V. Sridhar, G.H. Kulkarni,(2010)“4-Bit Flash Analog to Digital Converter Design using CMOS-LTE Comparator", IEEE Asia Pacific Conference on Circuits and Systems (APCCAS) ,ISBN no. 978-1-4244-7456-1, pp.772-775.

[27] De-Shiuan Chiou, Yu-Ting Chen, Da-Cheng Juan, and Shih-Chieh, (2010) "Sleep Transistor Sizing for Leakage Power Minimization Considering Temporal Correlation”, IEEE transactions on computer-aided design of integrated circuits and systems, Vol. 29, no. 8, pp.1285-1290.

[28] R. Jacob Baker (2009) “CMOS: Mixed Signal Circuit Design”, Second Edition, John Wiley \& Sons, ISBN 978 0470-29026-2.

[29] A.B. Bhattacharyya (2009) “Compact MOSFET Models For VLSI Design”, First Edition John Wiley \& Sons, ISBN: 978-0-470-82342-2.

[30] P.Iyappan, P.Jamuna and S.Vijayasamundiswary, (2009) "Design of Analog to Digital Converter Using CMOS Logic", IEEE International Conference on Advances in Recent Technologies in Communication and Computing, ISBN no. 978-0-7695-3845-7, pp. 74-76.

[31] Erik Sall and Mark Vesterbacka,(2007) "Thermometer-to-Binary Decoders for Flash Analog-toDigital Converters", European Conference on Circuit Theory and Design (ECCTD), ISBN no.1-42441342-7, pp. 240-243.

[32] Mingzhen Wang, Chien-In Henry Chen, and Shailesh Radhakrishnan, (2007)“Low-Power 4-b 2.5GSPS Pipelined Flash Analog-to-Digital Converter in 130-nm CMOS”, IEEE Transactions on Instrumentation and Measurement, Vol. 56, no. 3, pp. 1064- 1073.

[33] Kaijian Shi David Howard ,(2006) "Challenges in Sleep Transistor Design and Implementation in Low-Power Designs” ACM transactions on DAC, ISBN no. 1-59593-381-6, pp.113-116.

[34] Jincheol Yoo,Kyusun Choi and Jahan Ghaznavi, (2003)“A A0.07 $\mu$ m CMOS Flash Analog-to-Digital Converter for High Speed and Low Voltage Applications”, ACM transactions on GLSVLSI, ISBN no. 1-58113-677-3, pp. 56-59.

[35] Xuemei (Jane) Xi, Mohan Dunga, Jin He, Weidong Liu, Kanyu M. Cao, Xiaodong Jin, Jeff Jou, Mansun Chan, Ali M. Niknejad, Chenming Hu, (2003)“BSIM4.3.0 MOSFET Model: User Manual”.

[36] P. E. Allen and D. R. Holberg (2002) CMOS Analog Circuit Design, second edition oxford university press. ISBN no. 0195116445.

[37] D. Lee, J. Yoo, and K. Choi. (2002) "Design Method and Automation of Comparator Generation for Flash A/D Converters”. IEEE International Symposium on Quality Electronic Design, ISBN: 0-76951561-4, pp.138-142.

[38] D. Lee, J. Yoo, K. Choi, and J. Ghaznavi.(2002) "Fat Tree Encoder Design for Ultra-High Speed Flash A/D Converters", IEEE Midwest Symposium on Circuits and Systems, ISBN: 0-7803-7523-8, Vol. 2, No. 2, pp. 87-90.

[39] Pieter Harpe, Ming Ding, Ben Büsze, Cui Zhou, Kathleen Philips, Harmke de Groot (2013) "Nyquist AD Converters, Sensor Interfaces, and Robustness" Advances in Analog Circuit Design, Springer New York, ISBN 978-1-4614-4586-9.

[40] Skyler Weaver, Benjamin Hershberg, Pavan Kumar Hanumolu, Un-Ku Moon,(2012) "A multiplexerbased digital passive linear counter (PLINCO)" Analog Integrated Circuits and Signal Processing, Springer US, ISSN no. 1573-1979, Volume 73, Issue 1, pp 143-149. 
International Journal of VLSI design \& Communication Systems (VLSICS) Vol.4, No.3, June 2013

\section{Authors}

Arun Kumar Sunaniya is presently pursuing Ph.D from MANIT, Bhopal. He received B.E degree in Electrical Engineering from Government Engineering College, Ujjain (MP), India in 2002, M.Tech degree in Microelectronics \& VLSI Design from SGSITS, Indore (MP), India in 2008. He worked for IES/IPS Academy \& SDBCT at Indore. Presently he is working as Assistant Professor in department of Electronics and Communication Engineering at Corpoarate Group, Bhopal, India. He has five research publications on flash data converters in various international/national Journals \& conferences.

Kavita Khare received the B.E degree in electronics and communication engineering from Rani Durgavati Vishwavidyalaya, Jabalpur (MP), India in 1989, M.Tech degree in Digital Communication Systems in 1994 and $\mathrm{PhD}$ degree in the field of VLSI Design in 2004 She is working in MANIT, Bhopal since 1994 Presently she is working as Professor in Electronics and Communication Engineering department in MANIT, Bhopal. She has more than 150 publications in various international journals and conferences. She is also a Fellow of IETE (India) and Life Member of ISTE Journal.
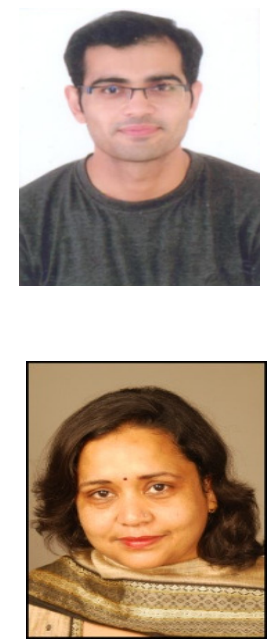